\title{
Efeitos da raquianestesia com bupivacaína hiperbárica $0,5 \%$ na pressão arterial média em cães induzidos e mantidos com propofol
}

\section{Subarachnoid anesthesia effects with hiperbaric bupivacaine $0,5 \%$ in the mean arterial pressure of dogs induced and maintened with propofol}

\author{
Isabella Dib Ferreira Gremião, ${ }^{\star \star}$ Paulo Roberto Loureiro do Nascimento, ${ }^{\star}$ Amary Nascimento Júnior, ${ }^{\star}$ João Henrique Neves

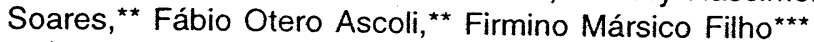

\begin{abstract}
Resumo
O presente estudo teve como objetivo avaliar alterações na pressão arterial média (PAM) com uso de bupivacaína hiperbárica $0,5 \%\left(0,3 \mathrm{mg} \cdot \mathrm{kg}^{-1}\right)$ no espaço subaracnóide de cães. Foram utilizados seis animais, fêmeas, sem raça definida, na faixa etária entre um a cinco anos, hígidas e com peso variando entre sete e $15 \mathrm{~kg}$. Após a contenção mecânica dos animais foi realizada a punção do ramo cranial da artéria safena com o intuito da aferição basal da pressão arterial média (PAM). Os animais foram pré-oxigenados com $\mathrm{O}_{2}$ a $100 \%$ (3 L/min) durante 5 minutos, induzidos com propofol $(6 \mathrm{mg} / \mathrm{kg})$ e, após a perda dos reflexos protetores, foi realizada a segunda aferição da PAM. A terceira mensuração da PAM procedeu-se após a intubação orotraqueal. Os animais foram monitorados e mantidos através de infusão contínua de propofol $\left(0,5 \mathrm{mg} \cdot \mathrm{kg}^{-1} \cdot \mathrm{min}\right)$ e a PAM mensurada durante 15 minutos em intervalos de 5 minutos. Posteriormente, foram realizadas a anti-sepsia e assepsia da região lombossacra € o espaço subaracnóide puncionado $\left(L_{6}-L_{7}\right)$ para administração de bupivacaína $\left(0.3 \mathrm{mg} \cdot \mathrm{kg}^{-1}\right)$. Uma vez constatado o bloqueio, a PAM foi mensurada durante um período de uma hora, com intervalos de 5 minutos. Como conclusão deste estudo não observamos nenhum valor pressórico compatível com um quadro hipotensivo.
\end{abstract}

Palavras-chave: raquianestesia, bupivacaína, cão, PAM.

\begin{abstract}
The purpose of this study was to evaluate the probable changes that occur in the mean arterial pressure (MAP) when hiperbaric bupivacaine $0,5 \%,(0,3 \mathrm{mg} / \mathrm{kg})$ is used in the subarachnoid space of dogs. Six healthy female mongrel dogs, with an average weight between seven and fifteen $\mathrm{kg}$ and age between one and five years were used. Initially, all the animal were phsysicaly restrainted and a catheter was introduced into the cranial branch of saphenous artery by a percutaneous procedure to the first measure of the MAP. Then, the animals were pre-oxygenated with oxygen-enriched air during 5 minutes and induced with propofol $(6 \mathrm{mg} / \mathrm{kg} \mathrm{IV})$. After the loss of protective reflexes, proceeded the second measure of the MAP. After the endotracheal intubation, proceeded the third measure of the MAP. The animal were monitored and during the maintenance with a continuous infusion of propofol $(0,5 \mathrm{mg} / \mathrm{kg} / \mathrm{min})$, the MAP was measured every five and ten minutes until fifteen minutes. Following a wide depilation and surgical scrub of the lumbsacral area, we introduced the spinal needle in the $L 6-L 7$ interspace for the administration of the bupivacaine. Once the subaracnoid anesthesia was accomplished, we measured the MAP every five minutes during one hour. This study allows us to observe that none of the patients developed hypotension.
\end{abstract}

Keywords: subarachnoid anesthesia, dog, MAP.

\section{Introdução}

A raquianestesia, também denominada anestesia espinhal, intratecal ou subaracnóidea, é aquela em que a solução anestésica é administrada diretamente no espaço subaracnóide, bloqueando temporariamente as raízes nervosas espinhais e os tecidos inervados pelos nervos bloqueados (Booth, 1992). Trata-se de uma técnica que permite obter anestesia de boa qualidade em área extensa do corpo, injetando-se dose muito pequena de anestésico 10-

\footnotetext{
* Professores da disciplina de Técnica Cirúrgica e Anestesiologia Veterinária - UFF.

* Alunos de mestrado em Clinica Médica e Cirurgia Veterinária - UFF.

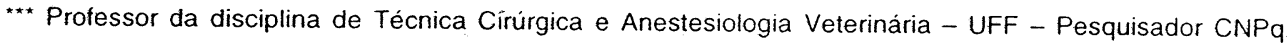

Rua Vital Brazil Filho, 64 - Santa Rosa - Niterói - RJ - CEP 24230-340 - e-mail: marsico@ nitnet.com.br
} 
cal (Belzarena e Domingues, 1995), estando indicada para cirurgias ábdominais, obstétricas e de membros posteriores (Souza, 1995). Dentre os anestésicos locais, os mais utilizados atualmente na raquianestesia são: lidocaína, bupivacaína e, mais recentemente, ropivacaína. Agentes opiáceos associados ou não aos anestésicos locais podem ser também utilizados neste tipo de técnica (Cousin e Mather 1984).

O cloridrato de bupivacaína é um anestésico local do tipo amida, um homólogo da mepivacaína, sintetizada em 1957 (Lumb e Jones, 1984). É um agente potente, capaz de produzir anestesia prolongada (Ritchie e Greene, 1994). A introdução da bupivacaína como agente anestésico para anestesia subaracnóidea foi associada a um novo interesse neste tipo de anestesia, principalmente devido a um aumento da duração de ação do bloqueio sensitivo, sem aumento dos efeitos colaterais e/ou toxicidade.

O propofol (2,6-diisopropilfenol) pertence ao grupo dos alquilfenólicos e apresenta propriedades hipnóticas e sedativas em animais (James e Glen, 1980). A via de administração preconizada é a venosa, podendo apresentar dor e desconforto durante a infusão. Todavia, não é relatada a ocorrência de flebite e trombose (Marshall e Longnecker, 1994). A dose para indução anestésica utilizada em cães sem medicação pré-anestésica (MPA) é de 6 a $8 \mathrm{mg} \cdot \mathrm{kg}^{-1}$, porém, em animais com MPA, varia de 2 a $4 \mathrm{mg}^{\mathrm{kg}} \mathrm{kg}^{-1} \mathrm{EV}$ (Thurmon et al., 1994). A dose de infusão recomendada situa-se entre 0,15 a $0,4 \mathrm{mg} \cdot \mathrm{kg}^{-1} \cdot \mathrm{min}^{-1}$, devendo ser administrada, previamente, uma dose de indução (Hall e Chambers, 1987).

A mais importante conseqüência da raquianestesia é a alteração da função cardiovascular com redução da pressão sangüínea, em decorrência do bloqueio simpático. Nas áreas simpaticamente inervadas, as artérias e arteríolas dilatam-se, determinando uma redução da resistência vascular periférica (RVP) e da pressão arterial (PA) (Ritchie e Greene, 1994). Por outro lado, a utilização do propofol como agente indutor e/ou de manutenção anestesiológica, seja por infusão contínua ou em bolus, produz também uma hipotensão primáxia devido a uma vasodilatação arterial e venosa (Ilkiw et al., 1992).

O presente trabalho teve como objetivo avaliar alterações na pressão arterial média (PAM) quando se utiliza a bupivacaína hiperbárica $0,5 \%$, na dose de $0,3 \mathrm{mg} \cdot \mathrm{kg}^{-1}$, no espaço subaracnóide de cães induzidos e mantidos com propofol.

\section{Material e métodos}

Segundo Imbelloni et al. (1988), a dose total de bupivacaína hiperbárica $0,5 \%$ preconizada para humanos é de $15 \mathrm{mg}$ $(3 \mathrm{~mL})$. Baseando-se nas técnicas anestesiológicas e doses para bloqueio espinhal em humanos, e devido à escassez de trabalhos em Medicina Veterinária relacionados às doses de bupivacaína por via intratecal em cães, realizou-se um pré-experimento utilizando-se em quatro animais, separadamente, as doses de 0,1,0,3 e $0,6 \mathrm{mg} / \mathrm{kg}^{-1}$. Optou-se pela dose intermediária pelo fato de apresentar um adequado bloqueio motor, sensitivo e menor incidência de efeitos simpáticos.
Para realização deste estudo, foram utilizados seis animais, fêmeas, sem raça definida (SRD), na faixa etária entre 1 e 5 anos, hígidos, com peso variando entre 7 e $15 \mathrm{~kg}$. Previamente a cada experimento os animais foram submetidos a jejum alimentar de 12 horas e hídrico de 2 horas. Após tricotomia e anti-sepsia das regiões lombossacra, face anteromedial dos membros anteriores e face anteromedial da região da articulação tibio-tarsiana, realizou-se a punção do ramo cranial da artéria safena (Ghoshal, 1986) com dispositivo intravenoso $22 G,{ }^{1}$ o qual foi conectado a uma linha arterial heparinizada, e esta, a um manômetro aneróide, ${ }^{2}$ objetivando a aferição basal da PAM. A veia cefálica foi cateterizada com dispositivo intravenoso 20G, para a administração de solução fisiológica $\mathrm{NaCl}$ a $0,9 \%$, no volume de $20 \mathrm{~mL} / \mathrm{kg} / \mathrm{h}$ ora. Os animais foram pré-oxigenados com $\mathrm{O}_{2}$ a $100 \%$ (3L/min) durante 5 minutos, induzidos com propofol ${ }^{3} \mathrm{em}$ bolus na dose de 6 mg.kg-1 EV (Thurmon et al., 1994). Após a perda dos refexos protetores foi realizada a segunda verificação da PAM, e após a intubação orotraqueal, a terceira mensuração. Além da mensuração da pressão arterial média, foram monitorados freqüência cardíaca, ${ }^{4}$ freqüência respiratória e capnometria ${ }^{5}$ e oximetria ${ }^{6}$. Os animais foram conectados a um circuito avalvular sem absorção de $\mathrm{CO}_{2}{ }^{7}$ mantidos em ventilação espontânea e, a cada 10 minutos, promoveu-se um suspiro. A veia cefálica contra-lateral foi puncionada com catéter intravenoso 20G, conectado a uma bomba de infusão contínua ${ }^{8}$ para a administração de propofol na velocidade de 0.5 mg. Kg-1 $\mathrm{min}^{-1}$. A PAM foi mensurada durante 15 minutos com intervalos de 5 minutos. Posteriormente foi realizado o isolamento asséptico da região lombossacra e a agulha espinhal tipo "Quincke" 22G introduzida no interespaço $L 6-L 7$. Após observação da saída de LCR (líquido cefalorraquidiano), a cabeça do animal foi colocada em um plano mais elevado, e a bupivacaína hiperbárica $0,5 \%{ }^{10}$ na dose de $0.3 \mathrm{mg} \cdot \mathrm{Kg}^{-1}$ administrada lentamente, na velocidade de $1 \mathrm{~mL}$ em 15 segundos.

Após 10 minutos, constatado o bloqueio anestésico através da obsenvação de flacidez da musculatura dos membros posteriores, relaxamento do esfíncter anal e perda de sensibilidade na região posterior, a PAM foi mensurada durante 60 minutos, com intervalos de 5 minutos. Os resultados obtidos foram submetidos a tratamento estatístico pelo teste nãoparamétrico de Kruskal-Wallis, onde $a=95$ (S.A.S).

\footnotetext{
1 Jelco - Ethicon

2 Pressurveil - Concept

${ }^{3}$ Diprivan - Lab. Wellcome/ZENECA

${ }^{4}$ Eletrocardioscópio - Biomedical Systems Inc. 404 Patient Monitor

${ }^{5}$ Analisador de gases exalados - BCl Intertnational Multigas Monitor 9100

${ }^{6}$ Puiso oxímetro - Ohmeda Mod 3700

${ }^{7} \mathrm{KT}-7$ Takaoka

${ }^{8}$ Digipump SR2000 - Digicare

9 Spinal BD

${ }^{10}$ Marcaina Pesada $0.5 \%$ - Astra
} 


\section{Resultados}

Os resultados estão expressos em tabelas.

Tabela 1: Variação média ('C) da pressão arterial média (PAM), da saturação de oxigênio ( $\mathrm{SpO}_{2}$ ), freqüência cardíaca $(\mathrm{FC})$, freqüência respiratória $(\mathrm{Fr})$ e capnometria $\left(\mathrm{ETCO}_{2}\right)$ em seis cães induzidos e mantidos com propofol. Letras diferentes na mesma linha diferem estastisticamente $(p<0,05)$ pelo teste de Kruskal-Wallis

\begin{tabular}{|c|c|c|c|c|c|c|c|c|}
\hline & Basal & Após indução & $\begin{array}{c}\text { Após } \\
\text { intubação }\end{array}$ & $\begin{array}{l}\text { Início da } \\
\text { manutenção }\end{array}$ & $\begin{array}{c}\text { Manutenção } \\
\text { T5 }\end{array}$ & $\begin{array}{c}\text { Manutenção } \\
\text { T10 }\end{array}$ & $\begin{array}{c}\text { Manutenção } \\
\text { T15 }\end{array}$ & $\begin{array}{l}\text { Após punção } \\
\text { do espaço } \\
\text { subaracnóide }\end{array}$ \\
\hline$\underset{(\mathrm{mmHg})}{\text { PAM }}$ & $115 \pm 9,0^{\mathrm{A}}$ & $96 \pm 12^{\mathrm{B}}$ & $122 \pm 3,0^{\mathrm{C}}$ & $93 \pm 9,0^{B}$ & $104 \pm 12^{A}$ & $105 \pm 16^{A}$ & $91 \pm 15^{\mathrm{B}}$ & $109 \pm 10^{A}$ \\
\hline $\begin{array}{l}\mathrm{SpO}_{2} \\
(\%)\end{array}$ & - & $98 \pm 1,0^{A}$ & $98 \pm 1,0^{A}$ & $98 \pm 1,0^{A}$ & $98 \pm 1,0^{A}$ & $98 \pm 1,0^{A}$ & $98 \pm 1,0^{\mathrm{A}}$ & $98 \pm 1,0^{A}$ \\
\hline $\begin{array}{c}\text { FC } \\
(\mathbf{b p m})\end{array}$ & $120 \pm 5,0^{\mathrm{A}}$ & $128 \pm 10^{\mathrm{B}}$ & $137 \pm 10^{B}$ & $122 \pm 6,0^{A}$ & $120 \pm 4,0^{A}$ & $119 \pm 6,0^{\mathrm{A}}$ & $119 \pm 6,0^{\mathrm{A}}$ & $128 \pm 13^{A}$ \\
\hline $\begin{array}{c}\mathrm{ETCO}_{2} \\
(\mathrm{mmHg})\end{array}$ & - & - & $40 \pm 3^{A}$ & $40 \pm 3^{A}$ & $41 \pm 2^{A}$ & $41 \pm 3^{A}$ & $42 \pm 2^{A}$ & $36 \pm 4^{B}$ \\
\hline $\begin{array}{c}\text { FR } \\
(\text { resp/min) }\end{array}$ & $30 \pm 6,0^{A}$ & $20 \pm 3,0^{B}$ & $18 \pm 3,0^{8}$ & $16 \pm 2,0^{B}$ & $16 \pm 3,0^{B}$ & $14 \pm 3,0^{\mathrm{C}}$ & $12 \pm 4,0^{C}$ & $25 \pm 3,0^{A}$ \\
\hline
\end{tabular}

Tabela 2: Variação média ('C) da pressão arterial média (PAM), da saturação de oxigênio $\left(\mathrm{SpO}_{2}\right)$, freqüência cardíaca $(\mathrm{Fc})$, freqüência respiratória $(\mathrm{Fr})$ e capnometria $\left(\mathrm{ETCO}_{2}\right)$ durante sessenta minutos em seis cães após a instalação da raquianesteșia. Letras diferentes na mesma linha diferem estastisticamente $(p<0,05)$ pelo teste de Kruskal-Wallis

\begin{tabular}{|c|c|c|c|c|c|c|c|c|c|c|c|c|c|}
\hline & 0 & 5 & 10 & 15 & 20 & 25 & 30 & 35 & 40 & 45 & 50 & 55 & 60 \\
\hline $\begin{array}{c}\text { AM } \\
\text { mmHg) }\end{array}$ & $\begin{array}{c}98 \\
\pm \\
10^{B}\end{array}$ & $\begin{array}{c}88 \\
\pm \\
13^{\mathrm{B}}\end{array}$ & $\begin{array}{c}89 \\
\pm \\
14^{B}\end{array}$ & $\begin{array}{c}88 \\
\pm \\
14^{B}\end{array}$ & $\begin{array}{c}87 \\
\pm \\
14^{B}\end{array}$ & $\begin{array}{c}87 \\
\pm \\
16^{B}\end{array}$ & $\begin{array}{c}86 \\
\pm \\
15^{B}\end{array}$ & $\begin{array}{c}87 \\
\pm \\
17^{\mathrm{B}}\end{array}$ & $\begin{array}{c}87 \\
\pm \\
19^{B}\end{array}$ & $\begin{array}{c}84 \\
\pm \\
17^{B}\end{array}$ & $\begin{array}{c}84 \\
\pm \\
16^{3}\end{array}$ & $\begin{array}{c}84 \\
\pm \\
15^{B}\end{array}$ & $\begin{array}{c}84 \\
\pm \\
16^{8}\end{array}$ \\
\hline $\mathrm{SpO}_{2}$ & 98 & 98 & 97 & 97 & 98 & 98 & 98 & 98 & 98 & 98 & 98 & 98 & 98 \\
\hline (\%) & $\stackrel{ \pm}{1,0^{A}}$ & $1,0^{\mathrm{A}}$ & $\pm \frac{ \pm}{1,0^{\mathrm{A}}}$ & $\pm \frac{ \pm}{1,0^{A}}$ & \pm & $\stackrel{ \pm}{ \pm, 0^{\mathrm{A}}}$ & $\stackrel{ \pm}{1,0^{A}}$ & $\frac{ \pm}{1,0^{A}}$ & $\stackrel{ \pm}{1,0^{\mathrm{A}}}$ & $\stackrel{ \pm}{1,0^{\mathrm{A}}}$ & $\underset{1,0^{\mathrm{A}}}{ \pm}$ & $\stackrel{ \pm}{ \pm}$ & \pm \\
\hline $\begin{array}{c}\mathbf{F C} \\
(\mathbf{b p m})\end{array}$ & $\begin{array}{c}119 \\
\pm\end{array}$ & $\begin{array}{c}117 \\
\pm\end{array}$ & $\begin{array}{c}118 \\
\pm\end{array}$ & $\begin{array}{c}107 \\
\pm\end{array}$ & $\begin{array}{l}109 \\
\pm\end{array}$ & $\begin{array}{c}107 \\
\pm\end{array}$ & $\begin{array}{c}106 \\
\pm\end{array}$ & $\begin{array}{c}107 \\
\pm\end{array}$ & $\begin{array}{l}107 \\
\pm\end{array}$ & $\begin{array}{c}108 \\
\pm\end{array}$ & $\begin{array}{c}107 \\
+\end{array}$ & $\begin{array}{c}106 \\
+\end{array}$ & $\begin{array}{c}107 \\
+\end{array}$ \\
\hline & $5,0^{A}$ & $5,0^{\mathrm{A}}$ & $4, \overline{0}^{\mathrm{A}}$ & $6,0^{B}$ & $5,0^{\mathrm{B}}$ & $4,0^{B}$ & $5,0^{\mathrm{B}}$ & $4,0^{\mathrm{B}}$ & $7,0^{B}$ & $6,0^{\mathrm{B}}$ & $5,0^{B}$ & $6,0^{B}$ & $5.0^{\mathrm{B}}$ \\
\hline $\mathrm{ETCO}_{2}$ & $\begin{array}{l}38 \\
+\end{array}$ & 39 & 39 & $\begin{array}{c}-40 \\
+\end{array}$ & $\begin{array}{r}41 \\
+\end{array}$ & 41 & 41 & 41 & 41 & 41 & 41 & 42 & 42 \\
\hline$(\mathbf{m m H g})$ & $2^{ \pm}$ & $2^{ \pm}$ & $3^{ \pm}$ & $\frac{ \pm}{3^{A}}$ & $\frac{ \pm}{2^{A}}$ & $\frac{ \pm}{3^{A}}$ & $2^{ \pm}$ & $\frac{ \pm}{2^{A}}$ & $\frac{ \pm}{3^{A}}$ & $2^{ \pm}$ & $\frac{ \pm}{3^{A}}$ & $2^{ \pm}$ & $\frac{ \pm}{2^{A}}$ \\
\hline FR & 22 & 20 & 20 & 16 & 14 & 14 & 14 & 14 & 13 & 13 & 12 & 13 & 13 \\
\hline (resp/min) & $\underset{4,0^{A}}{ \pm}$ & $3,0^{\mathrm{A}}$ & $2,0^{A}$ & $2 \pm 0^{A}$ & $2, \pm$ & $\underset{3,0^{B}}{ \pm}$ & $\stackrel{ \pm}{ \pm, 0^{B}}$ & $3,0^{B}$ & $\underset{3,0^{\mathrm{B}}}{ \pm}$ & $\begin{array}{c} \pm \\
3,0^{\mathrm{B}}\end{array}$ & $\stackrel{ \pm}{3,0^{B}}$ & $3,0^{\mathrm{B}}$ & $2,0^{\mathrm{B}}$ \\
\hline
\end{tabular}

a $0,4 \mathrm{mg} \cdot \mathrm{Kg}^{-1} \mathrm{~min}$ podem levar a quadros hipotensivos graves. Esta observação não foi constatada neste estudo, pois nenhum paciente apresentou valores pressóricos compatíveis com um quadro hipotensivo.

Com relação à freqüência cardiaca, observouse um aumento $(p>0,05)$ após a indução com o propofol e a intubação orotraqueal. No primeiro caso, este acréscimo deveu-se provavelmente a uma resposta reflexa à vasodilatação (Mársico et al., 1991; Branson e Gross, 1994). Já em relação à intubação orotraqueal, o acréscimo da freqüência parece estar associado a uma estimulação do sistema nervoso simpático que pode ocorrer durante este procedimento (Smith et al., 1993). Não obstante, Muir e Hubbel (1989) e Ezquerra e Casaús (1992) relatam a ocorrência de um efeito cronotrópico negativo e Sebel e Lowdon (1989) observam efeitos variáveis sobre a freqüência cardiaca, quando do uso do propofol.

Após a indução, observamos que os animais apresentaram uma diminuição da PAM ( $p<$ $0,05)$ estando este decréscimo de acordo com

\section{Discussão}

O propofol é um agente anestésico que proporciona uma anestesia com rápida indução e recuperação, além de apresentar pouco efeito acumulativo, mesmo quando administrado em bolus ou em infusão contínua em cães. Vários autores preconizam a dose de $0,4 \mathrm{mg} . \mathrm{Kg}^{-1} \mathrm{~min}^{-1} \mathrm{como}$ ideal para manutenção anestésica (Hall e Chambres, 1987; Robertson et al., 1992). No pré-experimento deste estudo utilizou-se esta dosagem de manutenção. Entretanto, para uma perfeita imobilização do animal e punção do espaço subaracnóide sem riscos de danos às meninges ou ao cordão medular, não demonstrou ser suficiente. Por este motivo, optou-se pela dose de $0,5 \mathrm{mg} \cdot \mathrm{Kg}^{-1} \mathrm{~min}$ que se mostrou adequada para 0 procedimento, bem como para manutenção anestesiológica, apesar de Hall e Chambres (1987) relatarem que doses superiores
- citado por Branson e Gross (1994), que relatam que o propofol pode determinar uma queda da PA através de uma vasodilatação arterial e venosa, além de deprimir a contratilidade do miocárdio. O grau de hipotensão é similar àquele determinado pelo tiopental sódico e, em geral, transitório devido ao curto tempo de ação da droga, e em muitas vezes, retorna aos valores basais com a intubação orotraqueal do paciente, afirmativa que corrobora com as observaçōes deste estudo, onde encontrou-se um aumento da PAM $(p<0,05)$ após a intubação, provavelmente devido a um estímulo do sistema nervoso simpático (Smith et al.,1993), sendo observado um retorno posterior dos valores pressóricos na manutenção anestesiológica (Branson e Gross, 1994).

A mais importante conseqüência da raquianestesia é a alteração da função cardiovascular com redução da pressão 
sangüínea, em decorrência do bloqueio simpático por ação do anestésico local em contato com as fibras pré-ganglionares que emergem da medula espinhal (Gouveia e Labrune, 1991). Esta diminuição da PAM pode tornar-se mais acentuada com a utilização do propofol como agente de manutenção, devido à vasodilatação arterial e venosa (llkiw et al., 1992). De acordo com os resultados, observou-se uma diminuição gradativa e significativa $(p<0,05)$ da PAM após a instalação da raquianestesia, quando comparada com os valores pressóricos da manutenção anestesiológica somente com propofol. Esta adição do bloqueio primário do propofol com o bloqueio simpático do anestésico não foi suficiente para que

\section{Referências}

BELZARENA, S.D.; DOMINGUES, J.A. Raquianestesia: Anestésico local hiperbárico ou isobárico? In: CONGRESSO LUSO-BRASILEIRODE ANESTESIOLOGIA, 5/CONGRESSOBRASILEIRODEANESTESIOLOGIA, 42, 1995, Salvador (BA), Anais, p. 229.

BOOTH, N.H.; MCDONALD, L.E. Fammacologia e Terapêutica em Veterinária. 6. ed. Rio de Janeiro: Guanabara Koogan, 1992. Cap. 19: Anestésicos locais, p. 323-335; Cap. 20: Anestesia intratecal e epidural, p. 336-347.

BRANSON, K.R.; GROSS, M.E. Propofol in veterinary medicine. Journal of the American Veterinary Medical Association, v. 204, n. 12, p. 1888-1890, jun, 1994.

COUSINS, M.J.; MATHER, L.E. Intrathecal and epidural administration of opioids. Anesthesiology v. 61, p. 275-310, 1984.

EZQUERRA, L.J.; CASAÚS, J.U. Anestesia general mediante agentes inyectables. In: CALVO, E.L.J.; VALLES, M.A. Anestesia práctica de pequeños animales. Madrid: Mc Graw-Hill-Interamericana, 1992. Cap. 5, p. 75-96.

FAVAREL-GARRIGUES, J.F.; LASSIE, P.; THICOIPE, M.; DABADIE, P. Hemodynamic tolerance of spinal anesthesia in the elderly: comparision between single dose and continuous spinal anesthesia with bupivacaine. Anesthesiology, S1-A3, A 1022, 1994.

FELDMAN, H.S.; COVINO, B.G. A chronic model for investigation of experimental spinal anaesthesia in the dog. Anesthesiology, v. 54, p. 148-152, 1981.

GHOSHAL, N.G. Coração e artérias do carnivoro. In: Getty, R. Anatomia dos animais domésticos. 5. ed. Rio de Jăneiro: Guanabara Koogan, 1986. p. 1497-1549.

GOUVEA, M.A.; LABRUNIE, S.M. Complicações da Anestesia Regional. Revista Brasileira de Anestesiologia, São Paulo, v. 41, n. 1, p. 29-
37, jan/fev, 1991.

HALL, LW.; CHAMBERS, J.P. A clinical trial of propofol infusion anaesthesia in dogs. Journal of Small Animal Practice, v. 28, p. 623-637, 1987.

ILKIW, J.E.; PASCOW, P.J., HASKINS, S.C. Cardiovascular and respiratory effects of propofol administration in hipovolemic dogs. American Journal of Veterinary Research; v. 53, p. 2323-2327, 1992.

IMBELONI, L.E. Usando a raquianestesia. In: CONGRESSO LUSO-BRASILEIRO DE ANESTESIOLOGIA, 5/ CONGRESSO BRASILEIRO DE ANESTESIOLOGIA, 42, 1995, Salvador (BA), Anais, p. 227. os animais do nosso estudo apresentassem valores pres: sóricos pertinentes a um quadro hipotensivo.

\section{Conclusões}

Nas condições deste estudo, a raquianestesia com bupivacaína hiperbárica $0,5 \%$, na dose de $0,3 \mathrm{mg} / \mathrm{kg}^{-1}$, demonstrou ser uma técnica segura, principalmente em pacientes jovens e hígidos, embora de difícil realização, exigindo treinamento por parte do anestesiologista. Observou-se que, em nenhum momento, foi necessária reposição de volume extra ou administração de drogas vasopressoras e, tampouco pré-expansão volêmica como conduta profilática.

IMBELONI, L.E.; IPSEN, C.G.; MAIA, C.P. Anestesia subaracnóidea para RTU de próstata: Tetracaína comparada com bupivacaina. $R e-$ vista Brasileira de Anestesiologia, São Paulo, v. 38, n. 3, p. 185-188, maio/jun., 1988.

JAMES, R.; GLEN, J.B. Synthesis, biological evaluation and preliminary structure-activity considerations of a series of alkylphenols as intravenous anaesthetics agents. Journal of Medicinal Chemistry, $v$. 23, p. 1350-1357, 1980.

LUMB, W.V.; JONES, E.W. Veterinary Anesthesia. 2. ed. Philadelphia: Lea e Febiger, 1984. Cap. 15: Local anesthetic agents, p. 357-370.

MARSICO, F.; TENDILLO, F. J.; ALVAREZ, I. Un nuevo anestésico intravenoso: Propofol. Evaluación clínica y experimental. Clin. Peq. Animales, v. 11, p. 231-239, 1991.

MUIR, W.W.; HUBBELL, J.A.E. Handbook of Veterinary Anesthesia. 2. ed., St. Louis: Mosby, 1995. Cap. 6: Patient monitoring during anesthesia, p. 165-190.

RITCHIE, J.M.; GREENE, N.M. Anestésicos Locais. in: GILMAN, A.G. As bases farmacológicas da terapêutica. 8. ed. Rio de Janeiro: Editora Guanabara Koogan, 1994. p. 205-218.

ROBERTSON, S.A.; JOHNSTON, S.; BEEMSTERBOER, J. Cardiopulmonary, anesthetic and postanesthetic effects of intravenous infusions of propofol in greyhounds and non-greyhounds. American Journal of Veterinary Research, v. 53, p. 1027-1032, 1992.

SEBEL, P.; LOWDON, J.D. Propofol: a new intravenous anaesthetic agent. Anesthesiology, v. 71, p. 260-277, 1989.

SKARDA, R.T. Local and regional anesthetic and analgesic thechniques: dogs. In: LUMB, W. V.; JONES, E.W. Veterinary Anesthesia. 3rd ed. Baltimore: Willians e Wilkins, 1996, p. 426-445.

SMITH, J.A.; GAYNOR, J.S.; BEDNARSKI, R.M. Adverse effects of administration of propofol with various preanesthetic regimens in dogs. Journal of the American Veterinary Medical Association. v. 202, n. 7 , p. 1111-1115, 1993.

SOUZA, M.L.M. A crescente aceitação da raquianestesia. Anestesia Regional, São Paulo, 2. ed., n. 2, p. 2-4,1995.

THURMON, J.C.; KO, J.C.H.;BENSON, G.J.; TRANQUILU, W.J.; OLSON, W.A. Hemodynamic and analgesic effects of propofol infusion in medetomidina-premedicated dogs. American Journal of Veterinary Research, v. 55, n. 3, p. 363-367, 1994. 